\title{
Higher Order Suppressor (HOS) for the PolLux Microspectroscope Beamline at the Swiss Light Source SLS
}

\author{
U. Frommherz ${ }^{\mathrm{a}}$, J. Raabe ${ }^{\mathrm{b}}$, B. Watts ${ }^{\mathrm{b}}$, R. Stefani ${ }^{\mathrm{a}}$, U. Ellenberger ${ }^{\mathrm{a}}$ \\ ${ }^{a}$ Paul Scherrer Institut, Division of Mechanical Engineering Sciences, 5232 Villigen PSI, Switzerland \\ ${ }^{b}$ Paul Scherrer Institut, Swiss Light Source, 5232 Villigen PSI, Switzerland
}

\begin{abstract}
The mechanical design and performance of a device to suppress higher orders of a spherical grating monochromator at a constant deviation angle is described. The higher order suppressor (HOS) is used for a scanning transmission x-ray microspectroscope beamline (PolLux) at a bending magnet of the Swiss Light Source (SLS). The instruments allow microspectroscopy in polymer science, of biological samples in the water window as well as the study of magnetic materials with circular or linear polarized light in a photon energy range of $200 \mathrm{eV}$ to $1400 \mathrm{eV}$. The HOS uses three mirrors acting as a low pass filter for soft x-rays to improve the absorption spectroscopy of carbon, oxygen and nitrogen 1s core levels. The successful installation and operation of the HOS located after the monochromator is reported. First results obtained using samples from materials research and environmental sciences exemplify the improved spectroscopy capabilities of the instrument.
\end{abstract}

Keywords: Microspectroscopy, Monochromator, Higher Order, Suppress.

PACS: 07.85.Tt, 07.10.Cm, 68.37.Yz.

\section{INTRODUCTION}

PolLux is a beamline at the Swiss Light Source to be used for scanning transmission x-ray microscopy (STXM). It has been in operation since summer 2006 and has seen various experiments performed successfully. PolLux uses linearly or circularly polarized x-rays from a bending magnet with an extended photon energy range of 200 to 1400 $\mathrm{eV}$ to give access to absorption edges of light elements (i.e. 1s electrons of carbon, nitrogen and oxygen), as major constituents in organic materials and of transition metals that constitute many magnetic materials. The instruments thus allow microspectroscopy in a wide range of fields, including polymer science biology in the water window and the study of magnetic materials [1]. This HOS is designed to be an additional component in the PolLux beamline without introducing a beam deflection or offset, so that it can easily be put into the beam or be taken out. Beamlines at other facilities are usually designed for a smaller energy range than $200 \mathrm{eV}$ to $1400 \mathrm{eV}$, where the higher orders are suppressed by choosing a suitable angle of incidence for one of the mirrors, respectively. Another possibility is to use different monochromator designs, with the disadvantage of higher costs and lower photon flux.

The beamline layout is shown in figure 1. X-rays from the Swiss Light Source are coupled out at the bending magnet. The toroidal mirror focuses the light source onto the entrance-slit and to the exit-slit, in the horizontal and the vertical directions, respectively. A spherical grating monochromator, consisting of two gratings with a constant deviation angle of $175^{\circ}$ in horizontal dispersion, is located between these two slits. The monochromator mechanics is a new design that has been developed and built in-house. The monochromator includes both a $300 / \mathrm{mm}$ and a $600 / \mathrm{mm}$ diffraction grating, respectively. Their characteristics have been described and calculated [2]. Besides the first order X-rays to be used for experiments there is a significant contribution of second and third orders of x-ray energies transmitted by the beamline [3]. At the end station, a Fresnel zone plate (FZP) focuses the soft x-ray light to a diffraction limited spot on the sample to achieve high spatial resolution. An order selecting aperture (OSA) which is moveable between the zone plate and sample provides maximum rejection of higher diffraction orders (both from the monochromator as well as from the zone plate) and maximum transmission of first-order light of the monochromator through its aperture (OSA) for a given wavelength [1]. However, because of the photon energy dependent focal length of a zone plate, i.e. the third order foci of the third order $\mathrm{x}$-rays (with three times of the 
energy and one third of the wavelength of first order x-rays) are focused at the same spot like first order x-rays. Second order foci, and hence second order x-rays are suppressed by the design of the zone plate itself. To further improve the ratio of first order to higher orders of x-ray, the higher order suppressor (HOS) has been added to the monochromator. The HOS is also of new design, developed and built in-house. Its characteristics and its functionality are presented in this paper.

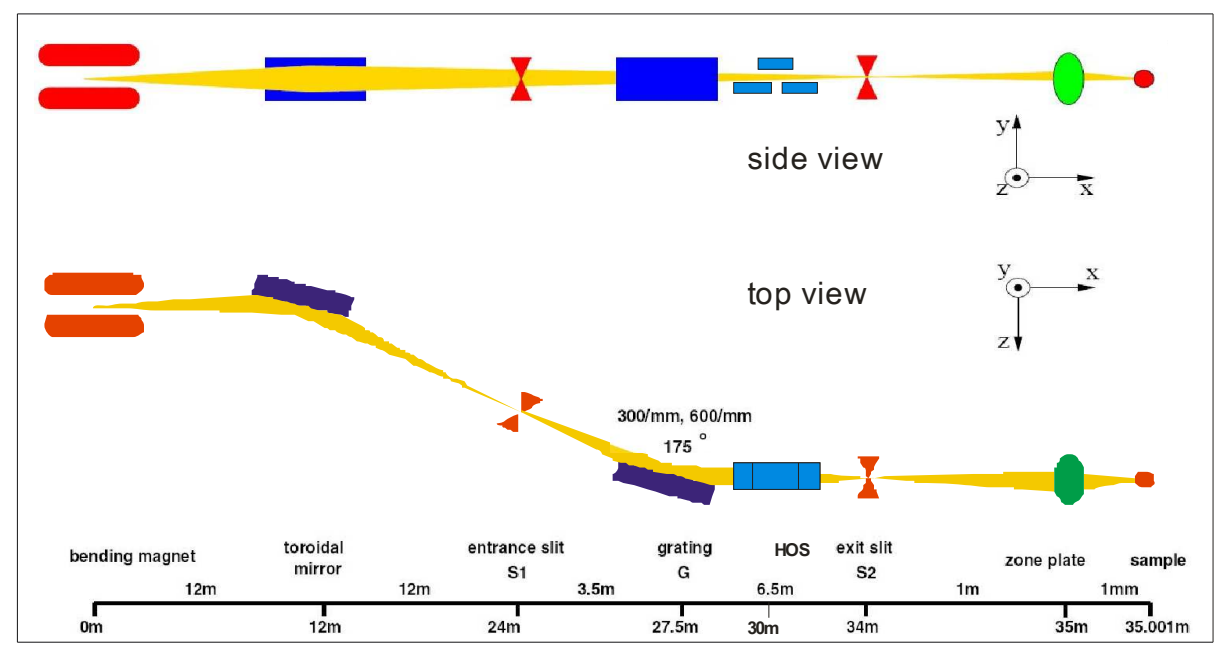

FIGURE 1. Layout of the PolLux beamline with higher order suppressor (HOS) located at $30 \mathrm{~m}$ after the bending magnet.

\section{CONCEPT OF MIRROR SYSTEM OF HOS}

The higher order suppression mirror system consists of a set of three fused silica mirrors with an $\mathrm{MgF}_{2}$ coating that provide stronger reflectivity of lower energy photons than higher energy x-ray photons. Higher order photons (i.e. photons with two or three times the principle energy) do not pass the HOS after three reflections and do not interfere with measurements made at lower energy absorption edges after the zone plate like $\mathrm{C}(300 \mathrm{eV}), \mathrm{N}(400 \mathrm{eV})$ or $\mathrm{O}(540 \mathrm{eV})$. As shown in figure 2 the set of three mirrors in the HOS are mounted in such a way that the x-ray beam trajectory remains unaltered in space despite the angle of reflection $[4,5]$. The top mirror remains stationary whereas the two lower mirrors are rotated symmetrically. The set of mirrors can pass the beam without reflection either by horizontal translation or by increasing the pitch of the mirrors until the beam passes between them.

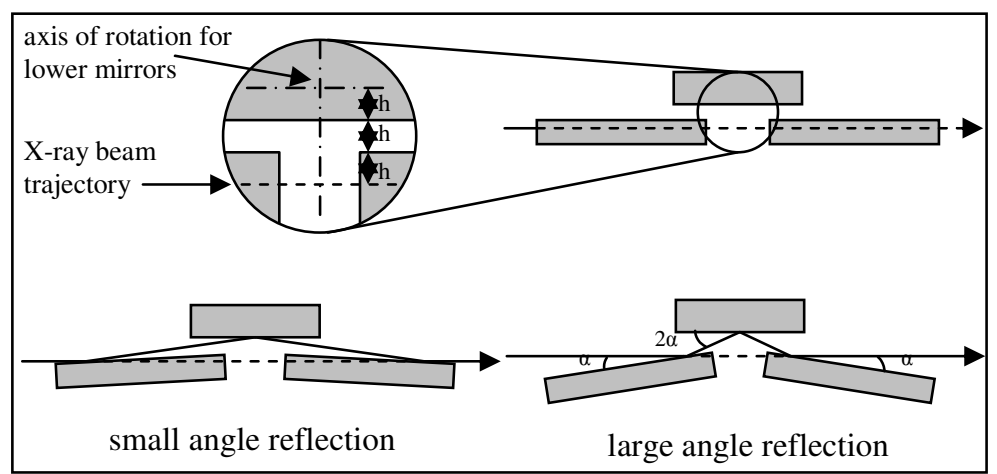

FIGURE 2. Mount of the three mirrors so that the $\mathrm{x}$-ray beam trajectory remains unaltered despite the angle of reflection.

As depicted in Fig. 3a at small angles of grazing incidence $\alpha\left(<1^{\circ}\right)$ the reflected x-ray photons significantly interact with the coating and higher orders of $x$-ray photons are transmitted as well. The cut-off energy at angle $\alpha$ of $1^{\circ}$ is at $700 \mathrm{eV}$ due to absorption by fluorine. At larger angles $\alpha$ of $2^{\circ}$, where higher orders of $\mathrm{x}$-ray photons are strongly suppressed, the cut-off energy decreases to $500 \mathrm{eV}$ due to absorption by oxygen in the substrate of the mirrors underneath the coating. The structure seen on the transmission spectra near $850 \mathrm{eV}$ is due to the Ni-coated gratings. The measured transmission performance of $\mathrm{HOS}$ has been compared to calculated data of $300 \mathrm{eV}$ 
(fundamental), $600 \mathrm{eV}$ ( $2^{\text {nd }}$ harmonic) and $900 \mathrm{eV}\left(3^{\text {rd }}\right.$ harmonic). Especially good agreement between measured and calculated data has been found for $300 \mathrm{eV}$. Suppression ratios of $300 \mathrm{eV}$ to $600 \mathrm{eV}$ and $300 \mathrm{eV}$ to $900 \mathrm{eV}$ have been calculated as well (graph and table in Fig. 3b). Suppression ratios of 1 to well above 1'000 have been obtained.

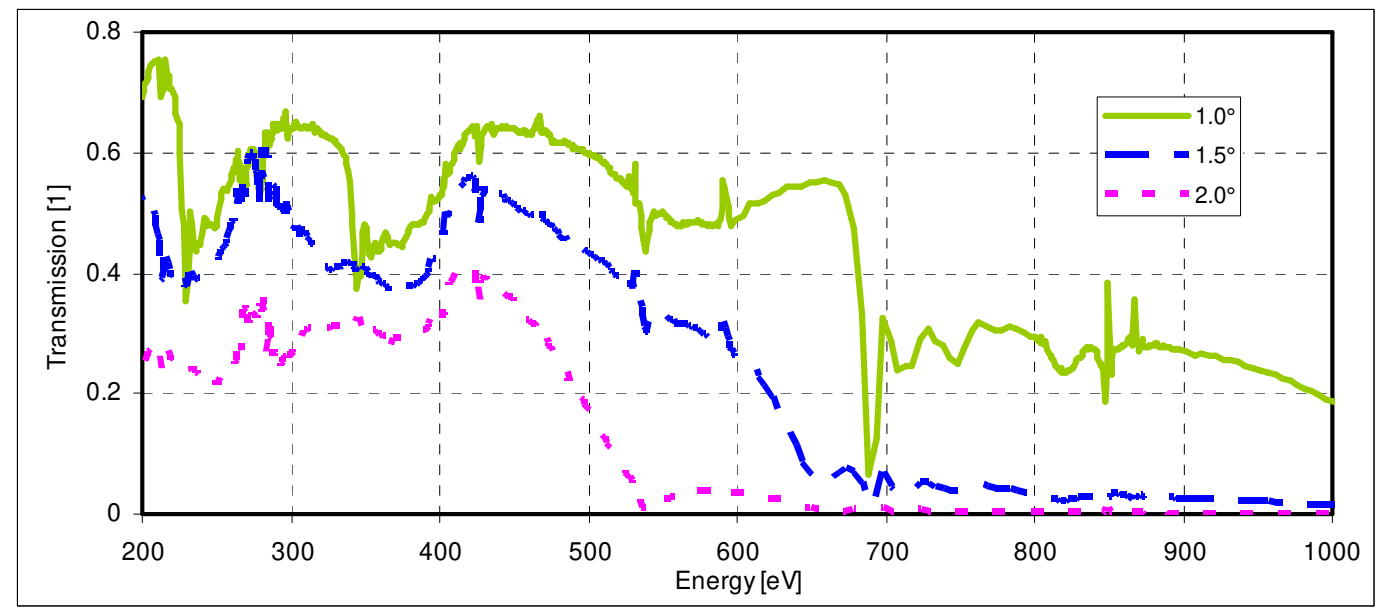

FIGURE 3A. Measurements of the transmission of the HOS mirror system as a function of energy. As the angle of incidence $\alpha$ is increased from $1^{\circ}$ to $2^{\circ}$ the cut-off energy changes from $700 \mathrm{eV}$ to $500 \mathrm{eV}$

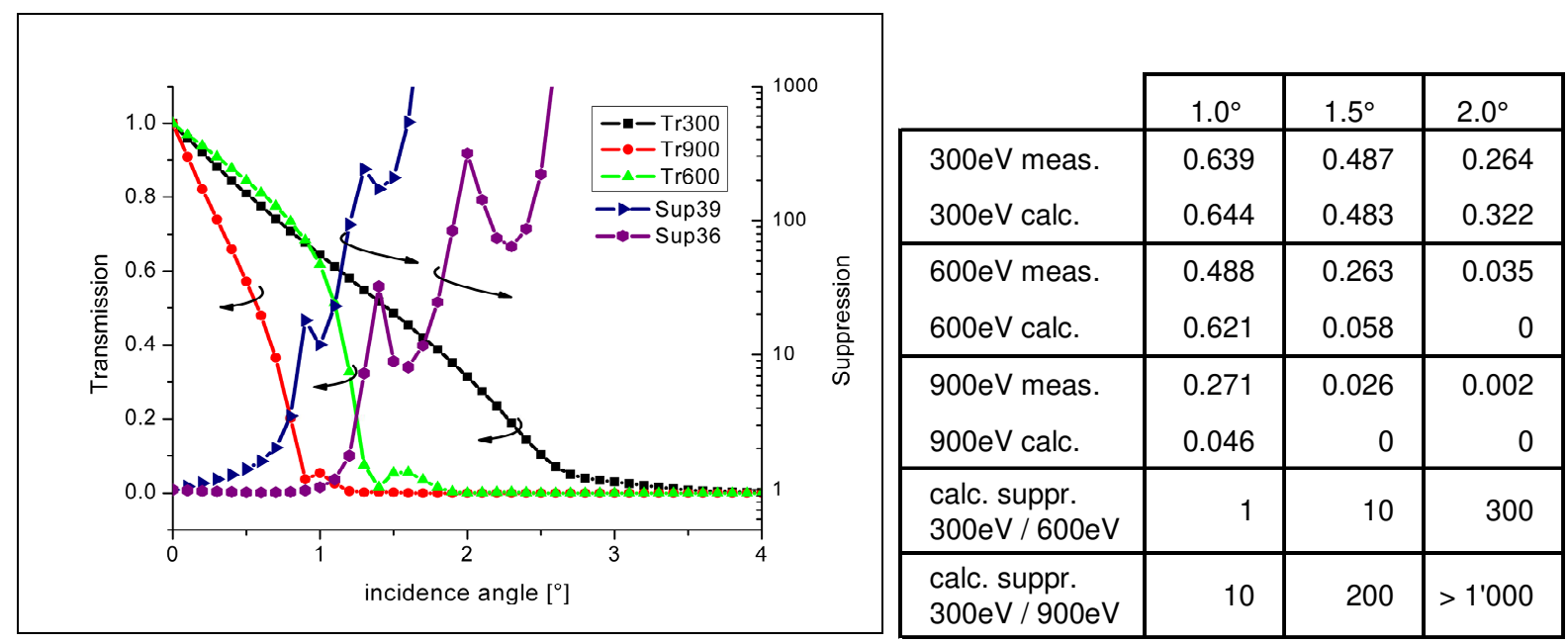

FIGURE 3B. Comparison of calculated data of transmitted energy (graph on left) to measured data (of Fig. 3a) is depicted in table (on right) as well as the calculated suppression ratios (see text for explanation)

\section{MECHANICAL DESIGN AND MANUFACTURING OF HOS}

Design and engineering of the HOS started in June 2008 and took 4 months. Manufacturing was performed between October 2008 and April 2009 by 2 professionally trained people (a work load equivalent of 12 personmonths). After installation and alignment one month later, on May 15th, 2009, first light was received in the PolLux beamline with the HOS inserted.

The HOS has 6 degrees of freedom with respect to the x-ray beam path. The vacuum chamber can be moved independently in three directions and can be rotated in two directions (pitch and roll). The precise pitch rotation is provided by the mirror system inside the vacuum chamber. The mirrors are rectangular and have clear apertures of $80 \mathrm{~mm} \times 40 \mathrm{~mm}$ (upper mirror) and $180 \times 40$ (lower mirrors) with respect to an x-ray beam diameter of 2 to $3 \mathrm{~mm}$ (see Fig. 4). The fused silica substrates are coated with $30 \mathrm{~nm}$ of $\mathrm{MgF}_{2}$. They have a surface roughness < $1.0 \mathrm{~nm}$ (rms) and slope errors of $0.5 \mu \mathrm{rad}$ (tangential) and $2.5 \mu \mathrm{rad}$ (sagital), respectively (rms). The manufacturer is Carl Zeiss Laser Optics GmbH in Germany. 


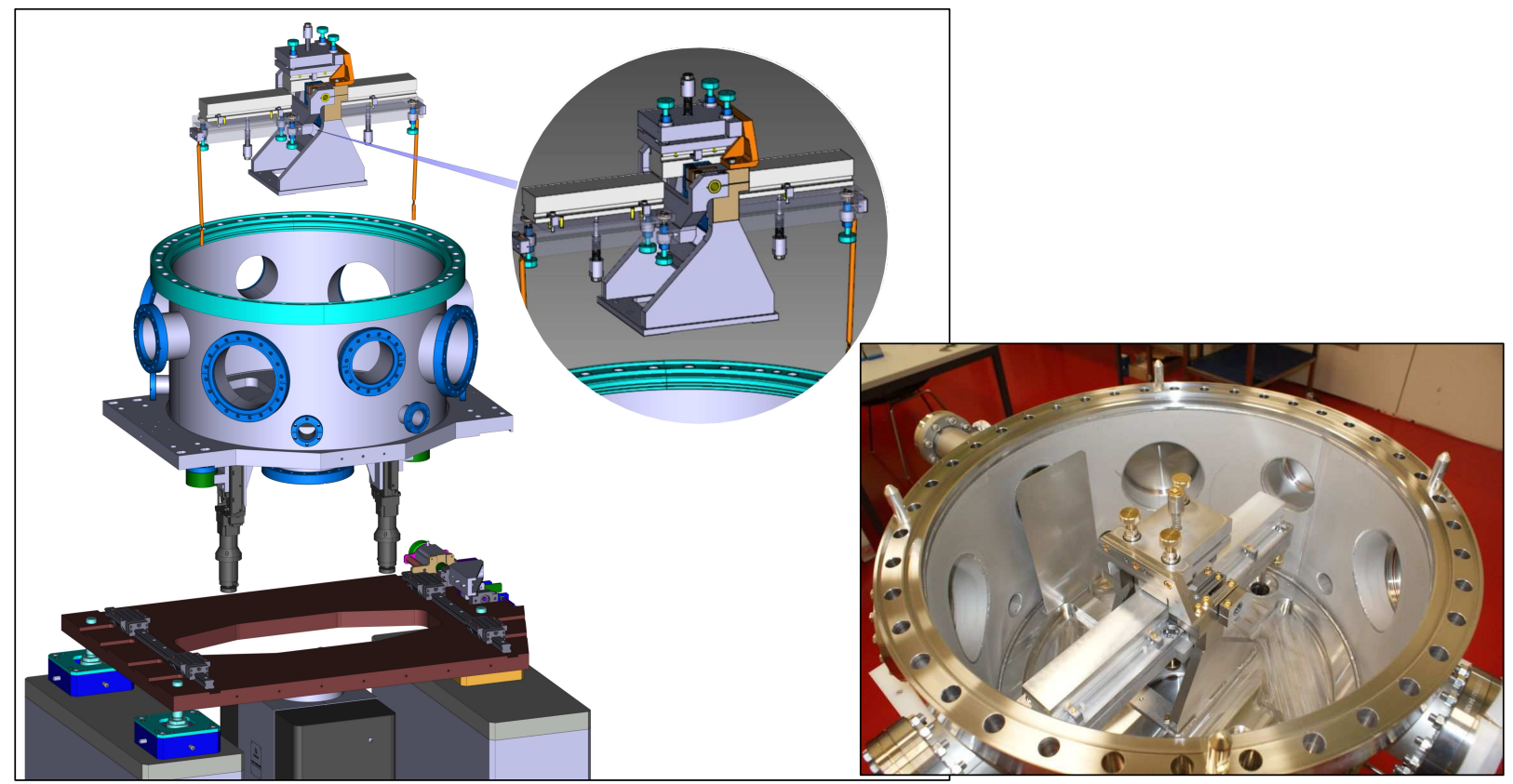

FIGURE 4. 3D-CAD drawing (Catia V5) of the HOS open (left) and the manufactured HOS with the mirror system in its center (right). The lower two mirrors rotate about a central pin and their angular position is driven by the two vertical rods that are visible in orange in the CAD drawing.

Carbon (C) deposits on mirrors may block the $\mathrm{x}$-ray beam. X-ray photons lead to secondary electron emission that heat residual $\mathrm{C}$ in vacuum which is then deposited on cold surfaces (usually mirrors) close to the $\mathrm{x}$-ray beam. To solve this problem, good results were obtained with an initial plasma cleaning $\left(40 \% \mathrm{O}_{2}\right.$ and $60 \% \mathrm{Ar}$ for stabilization of plasma arc) of the closed vacuum chamber or with ozone cleaning of components (i.e. a zone plate, with $\mathrm{O}_{3}$ from $\mathrm{O}_{2}$ under UV light). In either case $\mathrm{C}$ is "cold burned" to $\mathrm{CO}$ and $\mathrm{CO}_{2}$ which is extracted from the vacuum chamber through the vacuum pumps. Bake out of the vacuum chamber $\left(120^{\circ} \mathrm{C}\right.$ within 1 week $)$ is provided by electrical heating elements welded on the outer surface of the vacuum chamber.

The three mirrors were aligned manually while the vacuum chamber was still open. Under vacuum conditions the upper mirror remains fixed whereas both lower mirrors can be rotated by two separate drive systems outside the vacuum chamber. Both mirrors are synchronously driven by the same amount at the same time. Only the pitch of each lower mirror is rotated whereas yaw and roll are fixed. The angle of incidence $\alpha$ is precise within $0.1^{\circ}$ but the reproducible readjustment of $\alpha$ is within $1 \mu \mathrm{rad}$ since the exit slit located $4 \mathrm{~m}$ after the HOS has a width of only 20 $\mu \mathrm{m}$. The precision of pitch rotation of the two lower mirrors is achieved by using a bar of $25 \mathrm{~mm}$ length with two flexible elements on both sides and by using two precise pivot bearings (Rockwell Collins, free of play, made of stainless steel 1.4006). One flexible end of the bar is connected to one movable mirror. The other flexible end is coupled through a bellow to a linear stage with an encoder (PLS 85 from Micos, free of play, precision of $0.1 \mu \mathrm{m}$ ) outside the vacuum chamber. The mirrors are rotated to that precision under vacuum conditions $\left(10^{-10} \mathrm{mbar}\right)$. The angle of incidence can go up to $7.5^{\circ}$ (calculated) without blocking the x-ray beam but usually lies between $1^{\circ}$ to $2^{\circ}$.

\section{REFERENCES}

1. J. Raabe, G. Tzvetkov, U. Flechsig, M. Böge, A. Jaggi, B. Sarafimov, M.G.C. Vernooij, T. Huthwelker, H. Ade, D. Kilcoyne, T. Tyliszczak, R.H. Fink and C. Quitmann, "PolLux: A new facility for soft x-ray Spectromicroscopy at the Swiss Light Source", Review of Scientific Instruments 79, 113704 (2008).

2. S. Henein, U. Frommherz, R. Betemps, H. Kalt, U. Flechsig and J. Raabe, „Mechanical Design of a spherical grating Monochromator fort he Microspectroscopy Beamline PolLux at the Swiss Light Source", Proc. SRI (2006)

3. U. Flechsig, C. Quitmann, J. Raabe, M. Böge, R. Fink and H. Ade, „The PolLux Microspectroscopy Beamline at the Swiss Light Source", Proc. SRI (2006).

4. Ashok V. Pimpale, S.K. Deshpande, and V.G. Bhide, "Design Considerations for the Rotation of a Plane Premirror of a Monochromator for Reflecting Synchrotron Radiation onto the Same Spot of the Dispersing Grating of the XUV Beamline", Applied Optics vol. 30, no. 13, 1591 (1991).

5. Fritz Riemer, "Device for Applying Radiation at Adjustable Angles", United States Patent no. 4,490,041, Dec. 25 , 1984 